\title{
Cytotoxicity of anticancer drugs and PJ-34 (poly(ADP-ribose)polymerase-1 (PARP-1) inhibitor) on HL-60 and Jurkat cells
}

\author{
Maciej Stępnik ${ }^{A, F}$, Sylwia Spryszyńska ${ }^{B, C}$, Anna Gorzkiewicz ${ }^{B, C}$, Magdalena Ferlińska ${ }^{B, C}$ \\ Department of Toxicology and Carcinogenesis, Nofer Institute of Occupational Medicine, Łódź, Poland \\ A - research concept and design; $B$ - collection and/or assembly of data; $C$ - data analysis and interpretation; \\ $D$ - writing the article; $E$ - critical revision of the article; $F$ - final approval of article
}

\section{Address for correspondence \\ Maciej Stępnik \\ E-mail: maciej.stepnik@imp.lodz.pl \\ Funding sources \\ These investigations were supported by grant IMP1.6/2011 from the Nofer Institute of Occupational Medicine.}

\section{Conflict of interest}

none declared

Received on August 8, 2015

Revised on November 10, 2015

Accepted on December 2, 2015

DOI

10.17219/acem/60848

\section{Copyright}

Copyright by Author(s)

This is an article distributed under the terms of the

Creative Commons Attribution Non-Commercial License

(http://creativecommons.org/licenses/by-nc-nd/4.0/)

\begin{abstract}
Background. The majority of the clinical trials with poly(ADP-ribose)polymerase-1 (PARP-1) inhibitors were conducted or are ongoing in patients with solid tumors, while trials with leukemia patients are less frequent. Surprisingly scarce data is available on the combinatory effects of PARP inhibitors with DNA damaging antitumor drugs in leukemic cells (primary cells or established lines).

Objectives. The aim of the present study was to assess the effect of PJ-34 (PARP-1 inhibitor) on the cytotoxicity of different antileukemic drugs with different DNA damaging mechanisms and potency (doxorubicin, etoposide, cytarabine and chlorambucil) in human leukemic Jurkat and HL-60 cells.

Material and methods. Different exposure scenarios were applied: 1) $72 \mathrm{~h} \mathrm{simultaneous} \mathrm{incubation} \mathrm{with}$ PJ-34 (2.5 or $5 \mu \mathrm{M}$ for Jurkat and HL-60 cells, respectively) and a drug used at a wide concentration range; 2) preincubation of the cells with PJ-34 for $24 \mathrm{~h}$ and then with a combination of PJ-34 + drug for an additional $48 \mathrm{~h} ; 3$ ) preincubation of the cells with the drug for $24 \mathrm{~h}$ with a subsequent incubation with a combination of PJ-34 + drug for an additional $48 \mathrm{~h}$. Cytotoxicity was assessed using a WST-1 reduction test.

Results. It was determined that PJ-34, when used in all 3 scenarios, did not induce any significant enhancement of cytotoxicity of the drugs either in Jurkat or in $\mathrm{HL}-60$ cells.

Conclusions. Although the results do not confirm the beneficial effects of PARP inhibition in combination treatment of the leukemic cells, we propose that future studies including an additional step with the inhibition of DNA repair by homologous recombination should provide promising results.
\end{abstract}

Key words: PJ-34, doxorubicin, cytarabine, chlorambucil, etoposide 
Poly(ADP-ribose) polymerases, also termed ADP-ribosyltransferases with diphtheria toxin homology (ARTDs) according to a new nomenclature, catalyze the polymerization of ADP-ribose units from $\mathrm{NAD}^{+}$on acceptor proteins, leading to the formation of linear or branched polymers of ADP-ribose. The PARP superfamily encompasses at least 18 enzymes involved in several biological processes, including transcriptional regulation, DNA repair, cell cycle regulation, hypoxic response, inflammation, spindle pole function, oncogene-related signaling and cell death. ${ }^{1}$ Poly(ADP-ribose)polymerase-1 (PARP-1), an abundant and ubiquitous enzyme, is the best characterized member of the family. It accounts for $80-90 \%$ of detectable poly(ADP-ribose) synthesis following DNA damage.

Recently, PARP inhibitors were shown to selectively target DNA double-strand break (DSB) repair-deficient breast cancer type $1 / 2$ susceptibility protein (BRCA1/2) null cells for killing. ${ }^{2,3}$ The general understanding of this phenomenon, called synthetic lethality (i.e. when inactivation of either of 2 genes alone allows cell viability but simultaneous inactivation of both genes causes cell death), is that after inhibition of PARP-1 (a component of the DNA single-strand break (SSB) repair machinery), unrepaired SSB lesions are converted into DNA DSB during DNA replication and require activation of homologous recombination (HR) repair proteins (e.g. BRCA1/2) for their resolution. Hence, BRCA $1 / 2$ functionally null tumor cells treated with a PARP inhibitor accumulate DNA DSB and undergo cellular death. Besides its major role in detecting SSB, PARP-1 was shown to bind to and assist the repair of other damaged DNA structures, including stalled replication forks and DSB, having an effect on both HR and non-homologous end-joining repair processes (NHEJ). Assuming a high propensity of tumor cells towards genome instability and the complexity of PARP-related DNA repair routes, it is not surprising that PARP-1 inhibitors have raised many expectations as potential clinical anti-tumor drugs. These expectations have led to many trials with different generation PARP inhibitors used in monotherapy or in combination with other drugs (http://clinicaltrials.gov). Interestingly, so far the majority of the trials have been conducted (or are ongoing) in patients with solid tumors, while leukemia, myeloproliferative disorders or other hematological malignancies were less common. This situation is reflected by relatively scarce data in scientific literature on the combinatory effects of PARP inhibitors with other DNA damaging antitumor drugs in leukemic cells (primary cells or established lines).

In our study, we decided to assess the influence of a well known PARP-1 inhibitor, PJ-34, on the cytotoxic effects of different antileukemic drugs showing different DNA damaging mechanisms and potency. To this end, we selected doxorubicin (DNA intercalation and inhibition of topoisomerase II, DNA and RNA polymerases, DNA alkylation, disruption of calcium homeostasis and generation of free radicals), etoposide (pure topoisomerase II inhibitor), cytarabine (antimetabolite incorporating into DNA and interfering with DNA and RNA synthesis) and chlorambucil (alkylating agent of the nitrogen mustard type). PJ-34 is a very potent PARP inhibitor with half maximal effective concentration (EC50) of $20 \mathrm{nM}$, which is 10,000 times lower than the EC50 of $3-\mathrm{AB}^{4}$. For screening purposes, we selected Jurkat and HL-60 cells, which are well established human leukemic in vitro models. Although using a simultaneous coincubation of cells with different agents is currently the most common practice, in the present study, different exposure scenarios were applied. To this end, two general assumptions were made: 1) to preincubate the cells with PJ-34 for a longer time $(24 \mathrm{~h})$ to develop not only PARP inhibition, but also other recently-postulated potential PARP-independent effects (e.g. changes of cell cycle distribution ${ }^{5,6}$ or activation of the cytoprotective phosphatidylinositol-3 kinase (PI3K)Akt pathway), and 2) to superimpose PARP inhibition on a fully-developed DNA damage and DNA damage response/repair, i.e. commencing the co-exposure to PJ-34 after $24 \mathrm{~h}$ of preincubation of the cells with a drug. 5,6

\section{Material and methods}

\section{Chemicals and reagents}

The doxorubicin was from Sequoia Research Products (\#SRP04660d), chlorambucil was purchased from Enzo Life Science (\#ALX-400-049-G001), Cell Proliferation Reagent WST-1 (4-[3-(4-iodophenyl)-2-(4-nitrophenyl)2H-5-tetrazolio]-1,3-benzene disulfonate, \#11644807001) was obtained from Roche, RPMI 1640 + GlutaMAX culture medium (\#61870) and Fetal Bovine Serum (\#10270106) were purchased from Life Technologies and the Mycoplasma Detection Kit - MycoProbe (\#CUL001B) was from R\&D Systems. All other chemicals including etoposide (\#E1383), cytarabine (\#C1768), PARP inhibitor VIII - PJ34 (\#P4365), penicillin-streptomycin (\#P0781) and trypsin-EDTA (\#T4049) were from Sigma Aldrich.

\section{Cell lines}

The human $\mathrm{T}$ cell leukemia cell line (Jurkat - DSMZ \#ACC 282) and the human acute myeloid leukemia cell line (HL-60 - DSMZ \#ACC 3) were obtained from Deutsche Sammlung von Mikroorganismen und Zellkulturen GmbH (Braunschweig, Germany).

The cells were cultured in suspension in RPMI 1640, supplemented with $10 \%$ heat-inactivated fetal bovine serum and antibiotics (penicillin $100 \mathrm{U} / \mathrm{mL}$ and $100 \mu \mathrm{g} / \mathrm{mL}$ streptomycin). The cells were incubated in a $5 \% \mathrm{CO}_{2}$ humidified atmosphere. They were screened for Mycoplasma spp. infection using a Mycoplasma Detection Kit. 


\section{Cytotoxicity assessment - WST-1 reduction test}

The cytotoxicity of doxorubicin, etoposide, cytarabine, chlorambucil and PJ-34 on Jurkat and HL-60 cells was measured using a colorimetric WST-1 reduction test. The assay is based on the conversion by viable cells of light red tetrazolium salt WST-1 to the yellow formazan derivative, whose optical density is measured spectrophotometrically.

In brief, Jurkat cells $\left(4 \times 10^{3}\right.$ cells per well $)$ or HL-60 cells $\left(1.5 \times 10^{3}\right.$ cells per well $)$ were seeded in $50 \mu \mathrm{L}$ into a 96-well plate (NUNC \#167008) and exposed to test substances added as a $\times 2$ concentrated solution in $50 \mu \mathrm{L}$ of RPMI, for 24,48 or $72 \mathrm{~h}$. Then $10 \mu \mathrm{L}$ of the WST- 1 reagent was added to each well and the microplate was placed at $37^{\circ} \mathrm{C}$ for $1.5-2 \mathrm{~h}$. After $1 \mathrm{~min}$ shaking, the optical density of the formazan product was determined using a Multiscan RC spectrophotometer (Labsystems Helsinki, Finland) with a $450 \mathrm{~nm}$ filter and $620 \mathrm{~nm}$ filter as a reference. The results were expressed as the percent of cell survival (OD of exposed vs OD of non-exposed cells (control)).

The effect of PAPR-1 inhibitor - PJ-34 on the cytotoxicity of doxorubicin, etoposide, cytarabine and chlorambucil on Jurkat and HL-60 cells was also studied. In these experiments the cells were exposed to a combination of a drug with PJ-34 (used at maximum non-cytotoxic concentration determined in preliminary experiments) for $72 \mathrm{~h}$, or they were preincubated with PJ-34 or with the drugs for $24 \mathrm{~h}$. After the preincubation, the cells were treated with a combination of drugs with the inhibitor for an additional $48 \mathrm{~h}$. At the end of the exposure, the viability of the cells was assessed in a WST-1 reduction test.

\section{Statistical analysis}

The data was expressed as the mean \pm SD from the indicated number of separate experiments. The Inhibitory Concentrations inducing 50\% decrease in viability (IC50) with Confidence Intervals (CI) were calculated using GraphPad Prism v. 6.01 for Windows (GraphPad Prism Software, Inc., USA). After log transformation, the model of nonlinear regression (log(inhibitor) vs normalized response - variable slope) was applied.

\section{Results}

The cytotoxicity of PJ-34 (PARP-1 inhibitor) and selected drugs in Jurkat and HL-60 cells after 24-, 48- and 72-h exposure.

During 72-h incubation, the PJ-34 inhibitor decreased cell survival in a dose-dependent manner (Fig. 1). A higher sensitivity of Jurkat cells was observed, i.e. calculated IC50 values for all time-points were 2 -fold less in comparison to HL-60 cells. Based on the results of further studies on the 72-h exposures, the PJ-34 concentration of $2.5 \mu \mathrm{M}$ and $5 \mu \mathrm{M}$ were selected for Jurkat and HL-60 cells, respectively.

The cell viability at these concentrations exceeded $70 \%$. Prior to any studies on the inhibitory effects of PJ-34, a thorough dose-response cytotoxicity analysis of selected antitumor drugs on both cell lines was conducted. The data (Fig. 2) suggests a rather diverse potency of the drugs, with DOX and CYT showing the highest cytotoxic activity. In most cases (except CYT), after $72 \mathrm{~h}$ of incubation, the HL-60 cells were 2- (CHL) to 5- (ETO) fold more sensitive to the drugs in comparison to Jurkat cells.

\section{Cytotoxicity of selected anti-cancer drugs in combination with PJ-34}

Assuming different effects of PJ-34 on drug cytotoxicity depending on the extent of developing cellular damage and DNA damage response, 3 models of PJ-34-drug coincubations were applied, i.e. 1) simultaneous co-incubation (scheme in Fig. 3), 2) 24-h preincubation with a drug or 3) 24-h preincubation with PJ-34 (scheme in Fig. 4). In each case, the cytotoxicity was determined after a total of $72 \mathrm{~h}$ of exposure.

Fig. 1. Viability of Jurkat and HL-60 cells after 24,48 or $72-h$ exposure to PJ-34. WST-1 reduction test $(n=3-4)$. For each exposure, PJ-34 IC50 value has been calculated

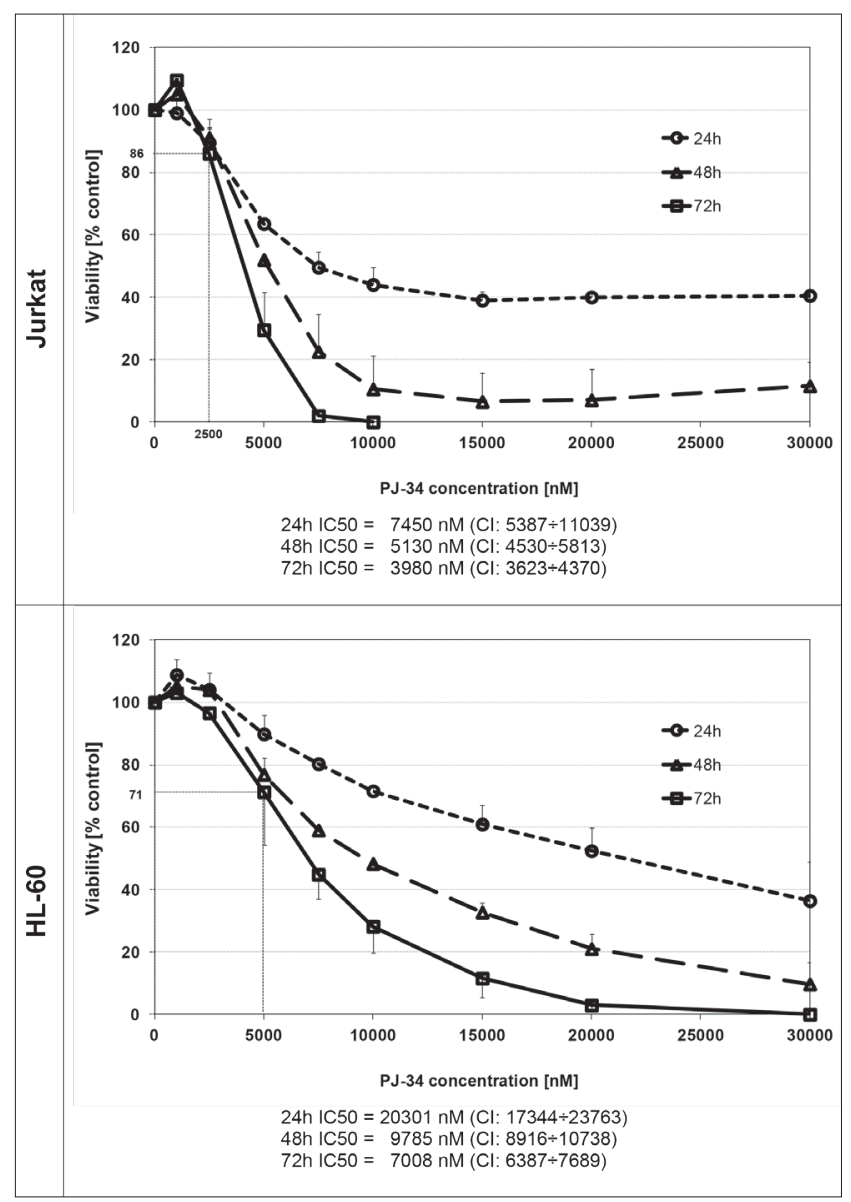


Fig. 2. Viability of Jurkat and HL-60 cells (\% control; the $\mathrm{Y}$ axis) after the exposure to selected anti-tumor drugs at the concentrations indicated (nM; the X axis): doxorubicin, cytarabine, etoposide or chlorambucil for $24 \mathrm{~h}$ (circles), $48 \mathrm{~h}$ (triangles) or $72 \mathrm{~h}$ (squares). WST-1 reduction test $(\mathrm{n}=3-4)$. For each exposure IC50 value has been calculated

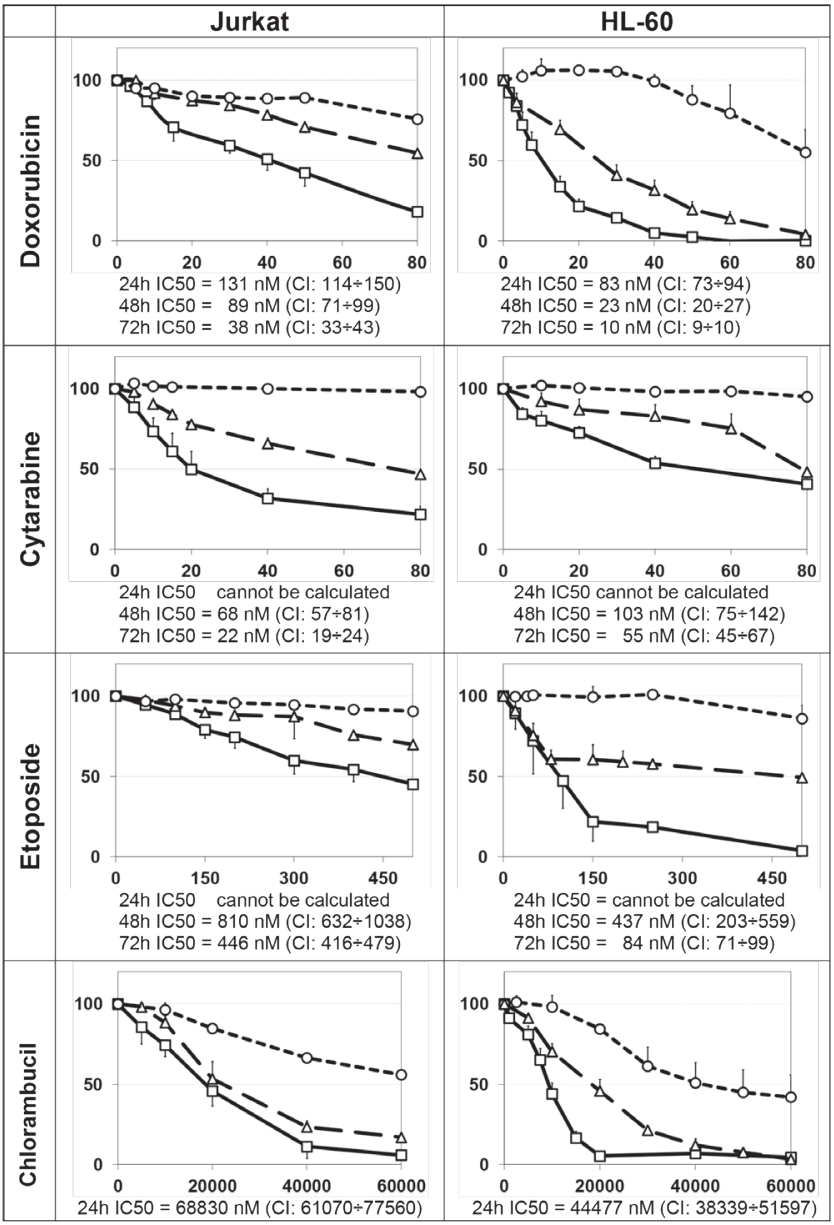

It was determined that PJ-34 when used simultaneously with the drugs did not induce any significant enhancement of cytotoxicity of the drugs either in Jurkat or in HL-60 cells (Fig. 5).

Similarly, PJ-34 did not significantly influence the cytotoxic potential of the drugs either when the cells were preincubated for $24 \mathrm{~h}$ with PJ-34 and then co-exposed with PJ-34 + drug for an additional 48 h (Jurkat cells: Fig. 6B; HL-60 cells: Fig. 7B) or when they were preincubated with a drug for $24 \mathrm{~h}$ and then co-exposed for an additional $48 \mathrm{~h}$ with drug-PJ-34 combination (Jurkat cells: Fig. 6A; HL-60 cells: Fig. 7A).

Fig. 3. Scheme of 72-h experiments - simultaneous incubation of Jurkat or $\mathrm{HL}-60$ cells with a single drug (green line) or combination of an anticancer drug with PJ-34 (red line)

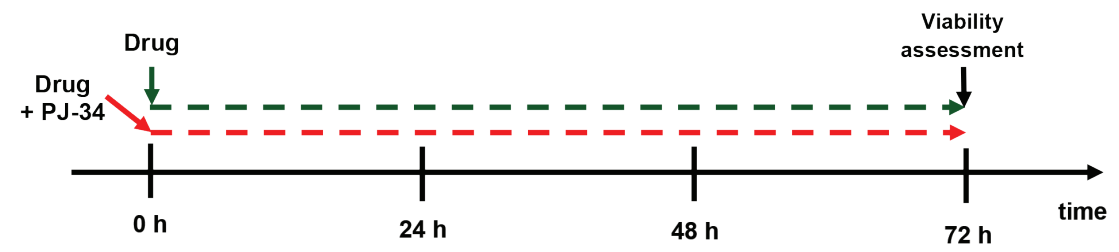

Fig. 5. Viability of Jurkat and HL-60 cells (\% control; the $Y$ axis) after the exposure to a combination of an anti-cancer drug ( $\mathrm{nM}$; the $X$ axis): doxorubicin, cytarabine, etoposide or chlorambucil with PJ-34 (at $2.5 \mu \mathrm{M}$ for Jurkat cells and $5 \mu \mathrm{M}$ for HL-60 cells) for $72 \mathrm{~h}$. WST-1 reduction test $(n=3-4)$. Squares - the drug, triangles - the combination of the drug with PJ-34. For each exposure IC50 value has been calculated

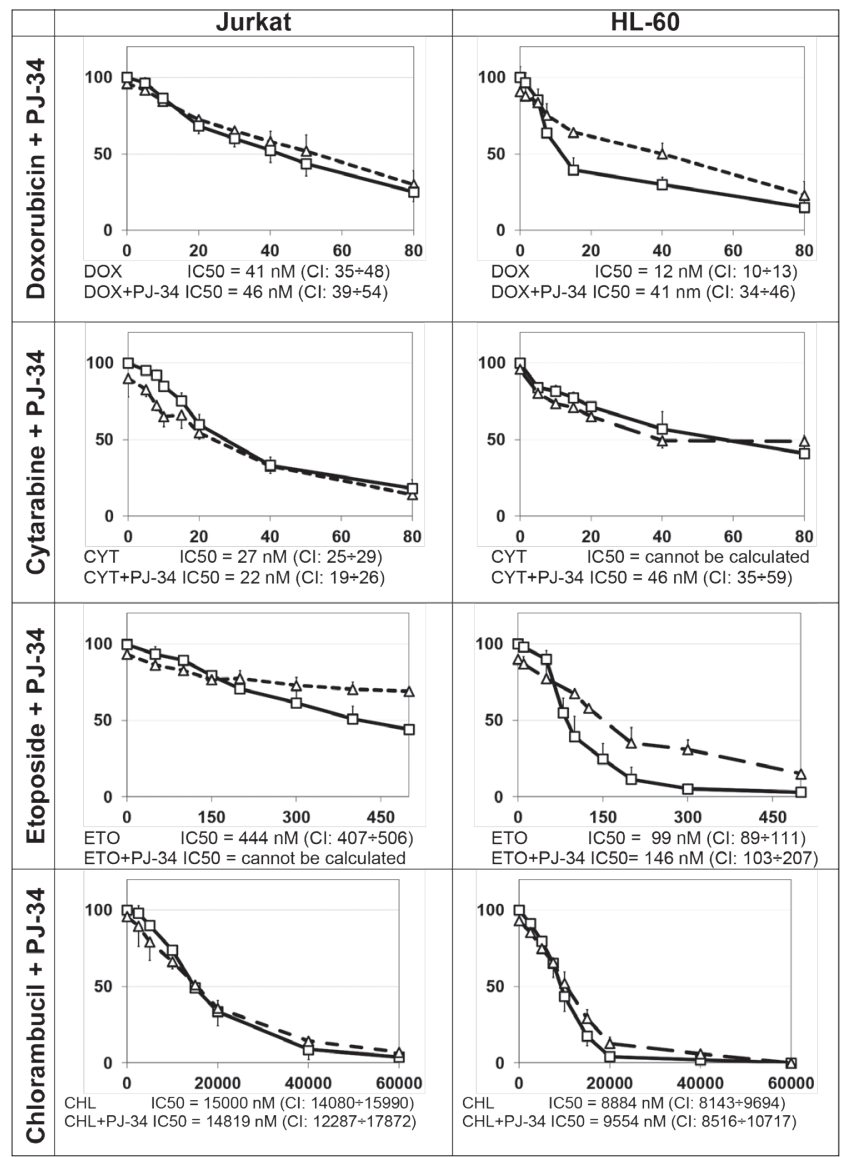

\section{Discussion}

In our study we hypothesized that increased DNA damage caused by the addition of PJ-34 to selected DNAdamaging drugs could produce a measurable increase in cytotoxicity in leukemic Jurkat and HL-60 cells. This concept is in line with current ideas of designing combined therapies where, by sensitizing tumor cells to cytotoxic agents, a lower dose could be given while maintaining the same relative efficacy and reducing the toxic side effects.

Although many reports indicate the usefulness of PARP-1 inhibitors in enhancing the cytotoxicity of DNA-damaging drugs in solid tumors, especially with BRCA $1 / 2$ deficiency, the experience with leukemic cells is relatively scarce. For many years, PARP-1 and PARP-2 have been recognized as central components of the Base Excision Repair/Singlestrand break repair process (BER). However, recently PARP-1 has been found to be activated by other types 
of lesions including DNA crosslinks, stalled replication forks and double-strand breaks. ${ }^{7}$ PARP-1 can bind to and be activated by DSB both in vitro and in vivo. ${ }^{8}$ It is predominantly involved in the HR-dependent repair of DSB at disrupted replication forks. While PARP-1 appears not to be involved in executing HR as such, some data indicates that it actively operates in the HR-dependent restart of stalled replication forks. ${ }^{9}$ The current model for PARP1-mediated replication fork stability assumes that if SSB results in fork collapse, resulting in a one-ended DSB and SSB in the sister chromatid, then PARP-1 binds the SSB and/or DSB and recruits XRCC1, thereby promoting the SSB repair process to repair the sister chromatid. ${ }^{10}$ PARP-1 may also repress $\mathrm{Ku} 70 / \mathrm{Ku} 80$ binding at the oneended DSB and thereby enabling HR-mediated template switching to promote fork
Fig. 4. Scheme of 24-h preincubation experiments. A) HL-60 or Jurkat cells were preincubated for $24 \mathrm{~h}$ with the drug, then for subsequent $48 \mathrm{~h}$ with a combination of the drug and PJ-34; B) HL-60 or Jurkat cells were preincubated for $24 \mathrm{~h}$ with PJ-34, then for subsequent $48 \mathrm{~h}$ with a combination of the drug and PJ-34

A.
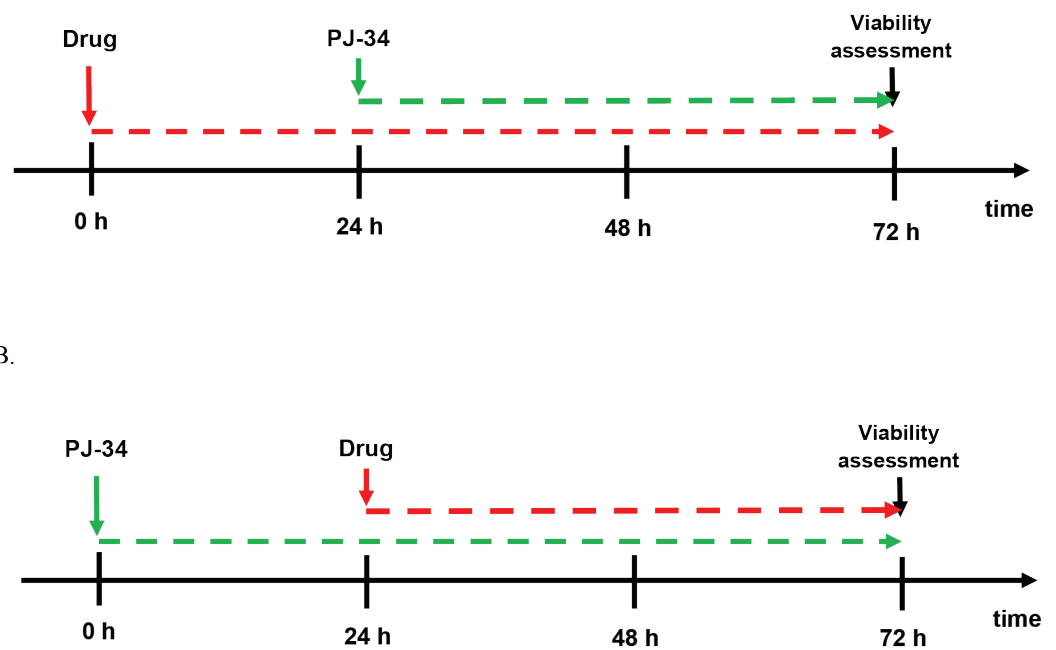

Table 1. Mechanisms of action and types of DNA repair induced by the selected drugs

Drug selected Mechanisms of action Type of repair of the DNA damage induced

\begin{tabular}{|c|c|c|}
\hline Doxorubicin (DOX) & $\begin{array}{l}\text { Topoisomerase II (Top2) inhibitor; "poisons" the enzyme by } \\
\text { stabilizing the DNA cleavage complexes, resulting in DNA } \\
\text { strand breaks }{ }^{21} \text { DNA intercalation, inhibition of DNA and RNA } \\
\text { polymerases, DNA alkylation, disruption of calcium homeostasis, } \\
\text { generation of free radicals; DOX-induced ROS generate mutagenic } \\
\text { base modifications and drive the formation of additional bulky } \\
\text { lesions in the form of DNA adducts and crosslinks, lesions which } \\
\text { cause replication fork stall and collapse; the higher cytotoxicity of } \\
\text { DOX compared to ETO may be due to a higher frequency of DNA } \\
\text { double strand breaks and/or by the formation of more persistent } \\
\text { cleavage complexes }\end{array}$ & $\begin{array}{l}\text { Two major pathways are active in the repair of DNA DSB: } \\
\text { NHEJ and HR repair; the phase of the cell cycle in which } \\
\text { DNA damage is induced is critical in determining which } \\
\text { of the response processes predominate: because HR } \\
\text { requires a homologous DNA sequence it is prevalent in } \\
\text { mitotic cells when a copy of the target DNA is available for }\end{array}$ \\
\hline Etoposide (ETO) & $\begin{array}{l}\text { Potent and the most selective Top2 cleavage complexes- } \\
\text { targeted drug currently in the clinic; does not intercalate DNA; } \\
\text { Top2 cleavage complexes produced form in a monotonic } \\
\text { manner without decrease at high drug concentration; the } \\
\text { complexes are readily reversible upon drug wash out, in contrast } \\
\text { to anthracyclines; despite the similar mechanisms of action of } \\
\text { ETO and DOX, the kinetics of cleavage complex formation and } \\
\text { recovery varies, the same as the ratio of single strand to double } \\
\text { strand Top2 mediated DNA breaks }{ }^{21}\end{array}$ & $\begin{array}{l}\text { exchange; DSB generated in the G1 phase of the cell cycle } \\
\text { are repaired by NHEJ }\end{array}$ \\
\hline Cytarabine (CYT) & $\begin{array}{l}\text { Primarily involves inhibiting DNA synthesis; after activation by } \\
\text { phosphorylation, the triphosphates of CYT are incorporated into } \\
\text { DNA opposite dG and inhibit DNA synthesis by stalling replication } \\
\text { forks; when incorporated into DNA, CYT is also a potent inhibitor } \\
\text { of topoisomerase I and II }\end{array}$ & $\begin{array}{l}\text { - repair mechanism not clear; nuclear co-localization } \\
\text { of Mre11, Rad50 and Nbs1 with phosphorylated ATM } \\
\text { and H2AX, increases in response to CYT; function of } \\
\text { ATM and MRN complex at sites of stalled replication } \\
\text { forks is unknown, but they may prevent fork collapse, } \\
\text { which otherwise could lead to DSB and chromosomal } \\
\text { aberrations }{ }^{24} \\
\text { - suggested DSB formed as a result of unresolved stalled } \\
\text { replication forks may be repaired by HR or NHEJ }\end{array}$ \\
\hline Chlorambucil (CHL) & $\begin{array}{l}\text { Most probably alkylates the nitrogenous bases of DNA (e.g. } \\
\text { formation of O6-chloroethylG, O4-chloroethylT adducts) and } \\
\text { forms inter- and intrastrand crosslinks; formation of crosslinks } \\
\text { results in uncoiling and twisting of the DNA helix } 22,23 \text { these } \\
\text { structural changes in the DNA duplex result in the inhibition of } \\
\text { DNA synthesis and DNA replication, DSB and finally cell death }\end{array}$ & $\begin{array}{l}\text { - primary chloroethyl adducts at O6-G are repaired by } \\
\text { direct base repair by O6-alkylG-DNA alkyltransferase } \\
\text { - highly cytotoxic interstrand crosslinks require nucleotide } \\
\text { excision repair (NER) factors (e.g. XPF-ERCC1) for incision } \\
\text { and HR or NHEJ to complete repair } \\
\text { - intrastrand crosslinks repaired by NER }\end{array}$ \\
\hline
\end{tabular}


Fig. 6. Viability of Jurkat cells (\% control; the Y axis) preincubated with an anti-cancer drug at the concentrations indicated ( $\mathrm{nM}$; the $\mathrm{X}$ axis) for $24 \mathrm{~h}$ (A) or with PJ-34 at $2.5 \mu \mathrm{M}$ (B) and then with the combination of both agents for subsequent $48 \mathrm{~h}$. WST-1 reduction test $(n=3)$. Squares - the drug, triangles - the combination of the drug with PJ-34. For each exposure IC50 value has been calculated

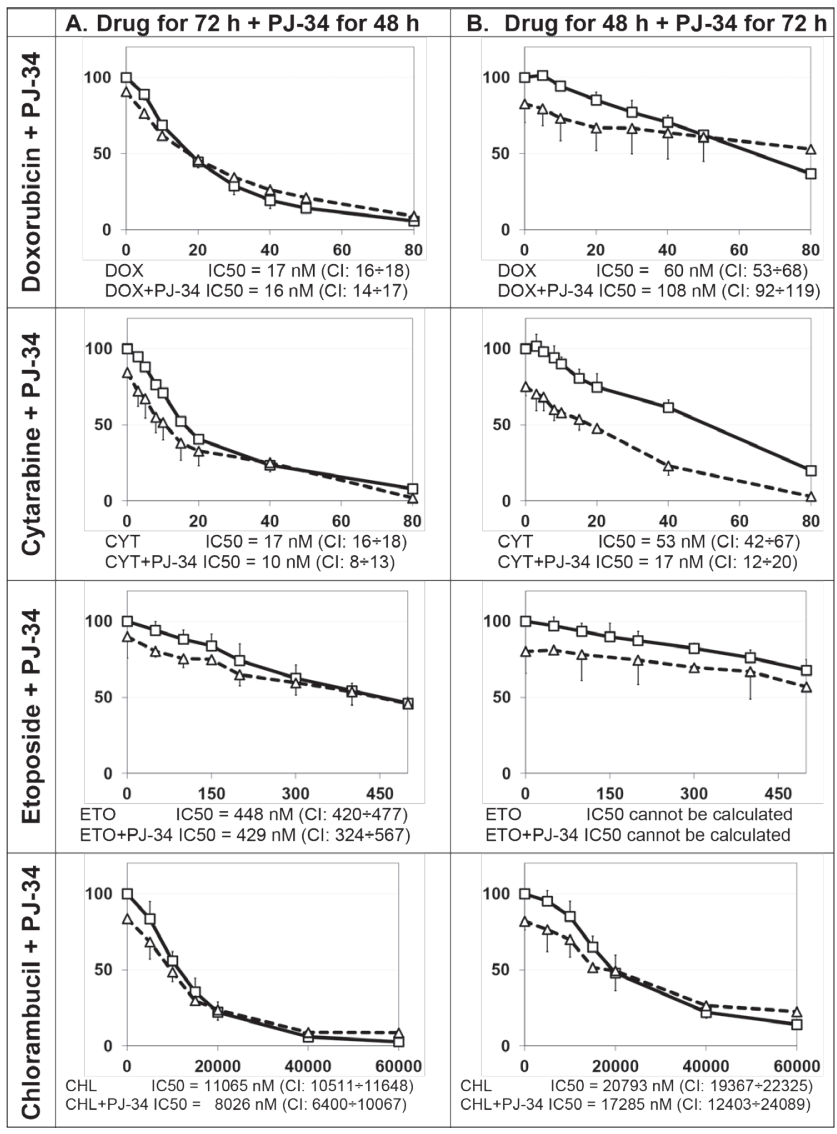

restart, preventing aberrant NHEJ repair of the DSB. PARP-1 may also promote HR directly, e.g. by regulating Mre11 nuclease activity at the DSB. ${ }^{11}$

The data quoted strongly suggests that PARP-1 inhibitors may be potentially useful in neoplasms defective in a vast array of DNA damage repair pathways, not only in BER (SSB repair) or HR (DSB repair). For this reason, in the present study, drugs with different putative modes of DNA-damaging activity were used (Table 1). Moreover, assuming an as-yet unidentified effect of PARP-1 inhibition depending on the timing of DNA damage induction (hence the extent of the damage and development of signaling of the damage), we applied 3 different exposure scenarios. Such approach is fully supported by many clinical trials with PARP inhibitors in combination with chemotherapy, where it is often difficult to define an optimum combination dose and schedule that would improve the therapeutic ratio.

In spite of the extended investigations, we did not observe any satisfactory effect of PJ-34 on the drugs cytotoxicity in either treatment schedules. Available literature data indicates rather divergent effects of PARP inhibition in leukemic cells, ranging from increased sensitivity to
Fig. 7. Viability of HL-60 cells (\% control; the Y axis) preincubated with an anti-cancer drug at the concentrations indicated ( $\mathrm{nM}$; the $\mathrm{X}$ axis) for $24 \mathrm{~h}$ (A) or with PJ-34 at $5 \mu \mathrm{M}$ (B) and then with the combination of both agents for subsequent $48 \mathrm{~h}$. WST-1 reduction test $(n=4)$. Squares - the drug, triangles - the combination of the drug with PJ-34. For each exposure IC50 value has been calculated

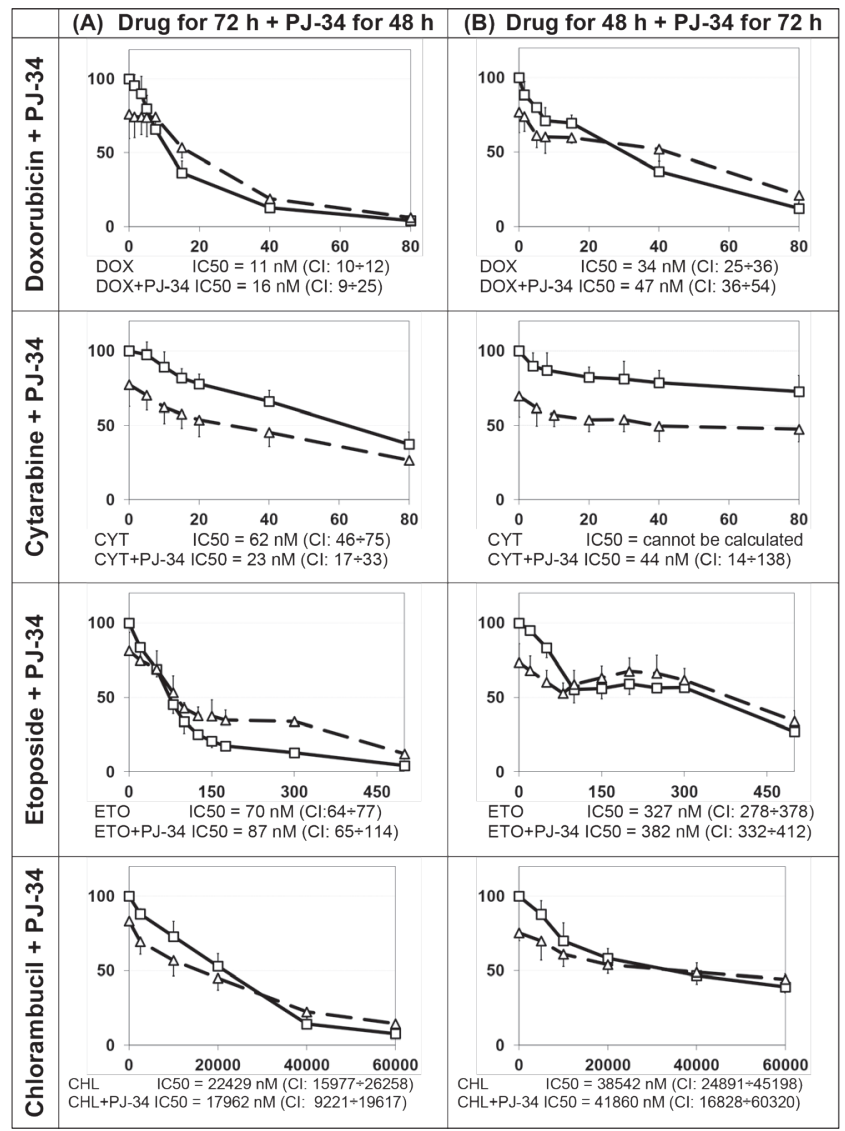

the drugs, through no effects, to even increased resistance of the cells. For example, 5'-aza-2'-deoxycytidine (a DNA methyltransferase inhibitor) failed to increase the cytotoxicity of PARP inhibitors (KU-0058948 and PJ-34); in contrast, MS275 (a histone deacetylase inhibitor) potentiated the cytotoxic effect of KU-0058948 and PJ-34 in all PARP inhibitor-sensitive leukemic cells. ${ }^{12}$ In human leukemia K562 cells, AG14361 (a PARP inhibitor) caused a 2-fold sensitization to camptothecin-induced cytotoxicity. ${ }^{13}$ CEP-8983 (a novel PARP inhibitor) synergized with bendamustine (a nitrogen mustard derivative) in killing primary chronic lymphocytic leukemia cells in vitro. ${ }^{14}$ Olaparib sensitized ATM null lymphoid tumor cells in vitro and in vivo to DNA-damaging agents. ${ }^{15}$ On the other hand, the pre-treatment of HL- 60 cells with 3 -aminobenzamide $(3-\mathrm{AB})$ or $6(5 \mathrm{H})$-phenanthridinone (PARP inhibitors), resulted in resistance to, rather than potentiation of, apoptotic death induced by DNA-damaging agents, idarubicin, etoposide and fludarabine. ${ }^{16}$ As can be seen, in spite of the very attractive hypothesis of the synthetic lethality and theoretical usefulness of PARP inhibitors in enhancing the DNA-damaging effects of antitumor drugs, the above-mentioned divergent results in 
leukemic cells are rather unexpected, with the underlying mechanisms probably being very complex.

The reasons for the lack of PJ-34 effects observed in the present study are unclear, however some potential explanations can be provided:

a) as we did not measure PARP activity, it cannot be excluded that the cells may have constitutively reduced protein expression, which may exert different effects compared to catalytic inactivation by PJ-34. Indeed, there are reports indicating that low PARP levels (and activity) attenuate responsiveness to PARP inhibitors. ${ }^{17}$ In this case, decreased PARP protein might be selectively advantageous to withstand the "poisoning" activity of drug-induced DNA-PARP aggregates. ${ }^{18}$ Quite similarly, the cytotoxic effect of topoisomerase inhibitors requires and is positively correlated with the levels and activity of topoisomerases; ${ }^{19}$

b) in our studies, we used the highest allowable concentration of PJ-34 which, after $72 \mathrm{~h}$, did not induce a significant cytotoxicity in the cells. However, again, it cannot be excluded that a potential residual activity of PARP-1 in PJ-34-treated cells might suffice for rescuing the drug's cytotoxic effects. Even if the PARP activity in the cells was not fully inhibited, we believe that such a condition much better reflects the clinical situation, where full inhibition of PARP-1 with currently-used inhibitors is impossible to achieve because of over-toxicity; ${ }^{20}$

c) the cells may have an efficient DNA repair capacity via different paths, which was sufficient to repair DNA damage after exposure to the drugs. For example, it is well recognized that cells deficient in DNA DSB repair are highly sensitive to the chemical inhibitors of PARP, however, cells with intact DNA DSB-response pathways repair damage with high fidelity and accordingly show very little sensitivity to PARP inhibitors.

\section{References}

1. Lupo B, Trusolino L. Inhibition of poly(ADP-ribosyl)ation in cancer: Old and new paradigms revisited. Biochim Biophys Acta. 2014;1846:201-215.

2. Audeh MW, Carmichael J, Penson RT, et al. Oral poly(ADP-ribose) polymerase inhibitor olaparib in patients with BRCA1 or BRCA2 mutations and recurrent ovarian cancer: A proof-of-concept trial. Lancet. 2010;376:245-251.

3. Tutt A, Robson M, Garber JE, et al. Oral poly(ADP-ribose) polymerase inhibitor olaparib in patients with BRCA1 or BRCA2 mutations and advanced breast cancer: A proof-of-concept trial. Lancet. 2010;376:235-244.

4. Garcia Soriano F, Virág L, Jagtap $\mathrm{P}$, et al. Diabetic endothelial dysfunction: The role of poly(ADP-ribose) polymerase activation. Nat Med. 2001;7:108-113.
5. Madison DL, Stauffer D, Lundblad JR. The PARP inhibitor PJ34 causes a PARP1-independent, p21 dependent mitotic arrest. DNA Repair. 2011;10:1003-1013.

6. Veres B, Gallyas F, Varbiro G, et al. Decrease of the inflammatory response and induction of the Akt/protein kinase $B$ pathway by poly-(ADP-ribose) polymerase 1 inhibitor in endotoxin-induced septic shock. Biochem Pharmacol. 2003;65:1373-1382.

7. Krishnakumar R, Kraus WL. The PARP side of the nucleus: Molecular actions, physiological outcomes, and clinical targets. Mol Cell. 2010;39:8-24.

8. Langelier MF, Planck JL, Roy S, Pascal JM. Structural basis for DNA damage-dependent poly(ADP-ribosyl)ation by human PARP-1. Science. 2012;336:728-732.

9. Beck C, Robert I, Reina-San-Martin B, Schreiber V, Dantzer F. Poly (ADP-ribose) polymerases in double-strand break repair: Focus on PARP1, PARP2 and PARP3. Exp Cell Res. 2014;329:18-25.

10. Caldecott KW. Protein ADP-ribosylation and the cellular response to DNA strand breaks. DNA Repair. 2014;19:108-113.

11. Bryant HE, Petermann E, Schultz N, et al. PARP is activated at stalled forks to mediate Mre11-dependent replication restart and recombination. EMBO J. 2009;28: 2601-2615.

12. Gaymes TJ, Shall S, MacPherson LJ, et al. Inhibitors of poly ADPribose polymerase (PARP) induce apoptosis of myeloid leukemic cells: Potential for therapy of myeloid leukemia and myelodysplastic syndromes. Haematologica. 2009;94:638-646.

13. Smith LM, Willmore E, Austin CA, Curtin NJ. The novel poly(ADPRibose) polymerase inhibitor, AG14361, sensitizes cells to topoisomerase I poisons by increasing the persistence of DNA strand breaks. Clin Cancer Res. 2005;11:8449-8457.

14. Dilley RL, Poh W, Gladstone DE, et al. Poly(ADP-ribose) polymerase inhibitor CEP-8983 synergizes with bendamustine in chronic lymphocytic leukemia cells in vitro. Leuk Res. 2014;38:411-417.

15. Weston VJ, Oldreive CE, Skowronska A, et al. The PARP inhibitor olaparib induces significant killing of ATM-deficient lymphoid tumor cells in vitro and in vivo. Blood. 2010;116:4578-4587.

16. Richardson DS, Allen PD, Kelsey SM, Newland AC. Effects of PARP inhibition on drug and Fas-induced apoptosis in leukaemic cells. Adv Exp Med Biol. 1999;457:267-279.

17. Farmer $\mathrm{H}, \mathrm{McC}$ abe $\mathrm{N}$, Lord $\mathrm{CJ}$, et al. Targeting the DNA repair defect in BRCA mutant cells as a therapeutic strategy. Nature. 2005;434:917-921.

18. Murai J, Huang SY, Das BB, et al. Trapping of PARP1 and PARP2 by Clinical PARP Inhibitors. Cancer Res. 2012;72:5588-5599.

19. Pommier Y. Drugging topoisomerases: Lessons and challenges. ACS Chem Biol. 2013;8:82-95.

20. Kummar S, Chen A, Ji J, et al. Phase I study of PARP inhibitor ABT888 in combination with topotecan in adults with refractory solid tumors and lymphomas. Cancer Res. 2011;71:5626-5634.

21. Pommier Y, Goldwasser F. Topoisomerase II inhibitors: The epipodophyllotoxins. In: Chabner BA, Longo DL, eds. Cancer Chemotherapy and Biotherapy: Principles and Practice. Philadelphia, USA: Lippincott Williams \& Wilkins.2011:392-410.

22. Mohamed D, Mowaka S, Thomale J, Linscheid MW. Chlorambuciladducts in DNA analyzed at the oligonucleotide level using HPLCESI MS. Chem Res Toxicol. 2009;8:1435-1446.

23. Charak S, Shandilya M, Tyagi G, Mehrotra R. Spectroscopic and molecular docking studies on chlorambucil interaction with DNA. Int J Biol Macromol. 2012;51:406-411.

24. Ewald B, Sampath D, Plunkett W. Nucleoside analogs: Molecular mechanisms signaling cell death. Oncogene. 2008;27:6522-6537. 